\title{
Cama de aviário e solo não autoclavados são eficientes no controle do crescimento de Scytalidium lignicola, causador da podridão negra da mandioca
}

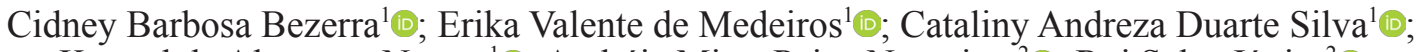

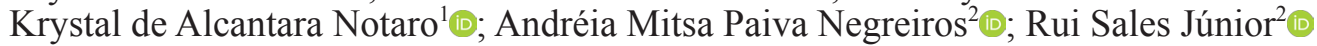

\begin{abstract}
${ }^{1}$ Universidade Federal Rural de Pernambuco (UFRPE), Unidade Acadêmica de Garanhuns, Garanhuns, Pernambuco, Brasil. ${ }^{2}$ Universidade Federal Rural do semiárido (UFERSA), departamento de Ciências Ambientais, Mossoró, Rio Grande do Norte, Brasil.

Autor para correspondência: Erika Valente de Medeiros (evmbio@gmail.com; evmlaw1@gmail.com).

Data de chegada: 26/10/2017. Aceito para publicação em:12/07/2018.
\end{abstract}

$10.1590 / 0100-5405 / 187102$

\section{RESUMO}

Bezerra, C.B.; Medeiros, E.V.; Silva, C.A.D.; Notaro, K.A.; Negreiros, A.M.P.; Sales Júnior, R. Cama de aviário e solo não autoclavados são eficientes no controle do crescimento de Scytalidium lignicola, causador da podridão negra da mandioca. Summa Phytopathologica, v.45, n.2, p.191-196, 2019.

A podridão negra da mandioca causada por Scytalidium lignicola vem se tornando uma doença importante para os estados produtores devido a grandes perdas de produção. Por isso o objetivo do presente trabalho foi avaliar o efeito de fontes e doses de matéria orgânica isolada e adicionada a solo arenoso sob a supressividade do crescimento micelial de $S$. lignicola CMM 1098. Foram realizados dois experimentos o primeiro foi visando avaliar o efeito dos extratos de materiais orgânico (cama de aviário, esterco caprino, bovino e húmus de minhoca) incorporados ao meio de cultura batatadextrose-ágar (BDA) nas concentrações (10, 20, 30 e 40\%) (v/v) e o segundo foi avaliar o efeito dos materiais orgânicos misturados com solo arenoso com as mesmas fontes e doses. A fonte cama de aviário foi a mais eficiente em inibir o crescimento micelial de $S$. lignicola a uma dose $40 \%$, com uma inibição do crescimento micelial de $69,75 \%$ apenas quando os extratos não foram autoclavados. Cama de aviário não autoclavado nas doses igual ou superior a 30\% foi eficiente na supressividade do crescimento micelial de $S$. lignicola CMM 1098. Solo arenoso não autoclavados adicionado à fontes e doses de matéria orgânica são altamente eficientes na supressividade do crescimento de S. linicola CMM 1098.

Palavras-chave: Manihot esculenta; esterco caprino; húmus de minhoca.

\section{ABSTRACT}

Bezerra, C.B.; Medeiros, E.V.; Silva, C.A.D.; Notaro, K.A.; Negreiros, A.M.P.; Sales Júnior, R. Non-autoclaved poultry litter and soil are efficient in controlling Scytalidium lignicola growth, the causal agent of cassava black root. Summa Phytopathologica, v.45, n.2, p.191-196, 2019.

Cassava root rot caused by Scytalidium lignicola has become an important disease for producing states due to large production losses. Thus, the aim of this study was to evaluate the effect of sources and levels of organic matter alone and added to sandy soil under suppressiveness to $S$. lignicola CMM 1098 mycelial growth. Two experiments were conducted, the first one was to evaluate the effect of organic extracts (poultry litter, goat manure, cattle and earthworm excrements) added to the culture medium potato dextrose agar (PDA) at different concentrations $(10,20,30$ and $40 \%)(\mathrm{v} / \mathrm{v})$, while the second experiment was to evaluate the effect of organic materials mixed with sandy soil, using the same sources and concentrations. The source poultry litter was most effective in inhibiting the mycelial growth of S. lignicola at a concentration of $40 \%$, inhibiting the mycelial growth by $69.75 \%$ only when extracts were not autoclaved. Non-autoclaved poultry litter at levels starting from $30 \%$ was efficient in suppressing S. lignicola CMM 1098 mycelial growth. Non-autoclaved sandy soil added to different sources and levels of organic matter is highly efficient for suppressiveness to S. lignicola CMM 1098 growth.

Keywords: Manihot esculenta; goat manure; earthworm excrements

Manihot esculenta Crantz vem ganhando importância no cenário mundial, pois é uma fonte de alimento para milhões de pessoas. É importante em diversos processos industriais (5), sendo uma das cinco commodities usadas na produção de bioetanol, biodíesel e biogás. Por isso a China vem estimulando o aumento de sua produção, pois há uma forte demanda por produtos derivados da mandioca (1).

Apesar de possuir um amplo espectro de aplicação, o cultivo da mandioca no Brasil é tradicionalmente realizado em um sistema de agricultura familiar na qual os agricultores geralmente utilizam material de propagação (manivas) de má qualidade fisiológica e sanitária devido à constante multiplicação. Dessa forma, essas manivas tornam-se, em muitos casos, uma importante fonte de disseminação de diversas doenças (18). Tudo isso se soma ainda à falta de um manejo cultural adequado que pode vir a contribuir para o aumento do número e intensidade de doenças.

A podridão negra da mandioca vem se tornando uma das principais doenças nos países produtores, como Brasil (20) e África (19). Trata-se de uma síndrome na qual pode ser causada por um ou mais patógenos, interagindo de forma isolada ou conjuntamente como Phytophthora drechsleri Tucker (16), Fusarium solani (3), Diplodia sp., Scytalidium lignicola (7) e Botriodiplodia sp..

Scytalidium lignicola Pesante é um fitopatógeno que causa a podridão negra em raízes e caules e vem causando perdas severas em áreas de produção de mandioca no Brasil (17). É uma doença de difícil 
controle, pois é um fungo habitante do solo que torna o uso de alguns produtos ineficientes quando aplicados ao solo e por não existirem ainda produtos registrados para essa doença, sendo necessários estudos de diferentes formas de manejo da doença.

Algumas estratégias de manejo desta doença são utilização de manivas de boa qualidade (18), rotação de culturas, cultivo consorciado, manipulação do solo para a indução da supressividade (3), uso de resíduos vegetais incorporados ao solo (12), Trichoderma como agente de controle biológico $(6 ; 7)$ e o uso da matéria orgânica $(20)$.

A aplicação de diversas fontes de matéria orgânica vem sendo estudada no manejo do patógeno como Phytophthora spp. (13) e Fusarium (15). sendo um forte aliado no controle de fitopatógenos, pois aumenta a atividade microbiana em diversos processos como; mecanismos de antibiose, hiperparasitismo, indução da resistência e competição por nutrientes, oxigênio e $\mathrm{CO}_{2}$, entre outros, melhorando as características químicas e físicas dos solos (11).

Devido à importância da doença em áreas produtoras de mandioca no mundo, associado a carência de trabalhos acerca de formas alternativas de manejo da doença causada por S. lignicola, este trabalho tem como objetivo avaliar o efeito de fontes e doses de matéria orgânica sem e com solo arenoso sobre a supressividade do crescimento micelial de S. lignicola.

\section{MATERIAIS E MÉTODOS}

\begin{abstract}
Obtenção do Scytalidium lignicola CMM 1098 e preparo do inóculo

O isolado de $S$. lignicola utilizado neste experimento foi proveniente de mandioca com sintomas de podridão radicular, coletado em fazenda produtora de mandioca no Município de Caetés-PE ( $8^{\circ} 46^{\prime} 22^{\prime}$ ' $\mathrm{S}$, $36^{\circ} 37^{\prime} 22^{\prime \prime} \mathrm{W}$ ) e havendo sido testado em estudo prévio e selecionado como o mais agressivo em teste de patogenicidade (17). Tal patógeno encontra-se depositado na coleção de cultura de fungos fitopatogênicos "PROF. MARIA MENEZES" (UFRPE), com o código CMM 1098.

O inóculo fúngico foi produzido em placa de Petri com meio de cultura BDA, na qual o isolado de $S$. lignicola CMM 1098 foi repicado e incubado por 14 dias em B.O.D. a $25 \pm 2^{\circ} \mathrm{C}$, sob alternância luminosa (12 h claro/ $12 \mathrm{~h}$ escuro).
\end{abstract}

Experimento 1: Avaliação do efeito de materiais orgânicos sobre o crescimento in vitro do fungo Scytalidium lignicola CMM 1098.

Neste experimento, foram utilizados diferentes fontes de resíduos orgânicos como; cama de aviário, esterco caprino, esterco bovino e húmus de minhoca selecionados pelo critério de disponibilidade na região. Esses materiais foram coletados na Clínica de bovinos da UFRPE e junto a produtores em Garanhuns,PE. Os mesmos foram postos para curtir ao sol, por um período de um mês acondicionados em bandejas, etc abertas?.

Desses resíduos foram obtidos extratos aquosos mediante mistura em volume do resíduo de cada material com água destilada na proporção 1:3 (p/v), deixando-o em repouso por uma hora. Duas formas de extração foram realizadas: uma sem e outra com autoclavagem $\left(120^{\circ} \mathrm{C}\right.$, $1 \mathrm{~atm}$ por $20 \mathrm{~min}$ ). Em ambos processos se procedeu a filtragem do extrato em papel filtro e armazenados em geladeira por $1 \mathrm{~h}$.

Após a obtenção dos extratos, os mesmos foram incorporados ao meio de cultura batata-dextrose-ágar (BDA) nas concentrações $(10,20$, 30 e 40\%) (v/v), sendo o pH corrigido para 6 mediante adição de NAOH ou $\mathrm{HCl}$. Posteriormente, os meios de cultura contendo o material foram vertidos em placas de Petri de $9 \mathrm{~cm}$ de diâmetro, onde após $24 \mathrm{~h}$ foram transferidos discos de BDA de $8 \mathrm{~mm}$ de diâmetro contendo o micélio de S. lignicola CMM 1098 depositadso na área central da palca de Petri.. Posteriormente as placas de Petri foram incubadas em estufa B.O.D. a $25^{\circ} \mathrm{C}$, de onde foram avaliadas diariamente quanto ao crescimento radial micelial, mediante a utilização de uma régua graduada em $\mathrm{mm}$.

$\mathrm{O}$ delineamento experimental aplicado ao ensaio foi inteiramente casualizado, distribuído em esquema fatorial $4 \times 5 \times 2+2$, sendo o primeiro fator materiais orgânicos: cama de aviário, esterco caprino, esterco bovino e húmus de minhoca; o segundo as concentrações: 0,10 , 20, 30 e 40\% o terceiro, autoclavagem ou não e mais dois controles: solo sem material orgânico com e sem autoclavagem, com quatro repetições. As variáveis analisadas foram:

- Taxa de crescimento micelial (TCM) em milímetro;

- Área abaixo da curva de crescimento micelial (AACCM), utilizando a fórmula, de acordo com a Eq. (1):

$$
A A C C M=\frac{\sum \dot{y}+(\dot{y}+1)}{2 . d_{t i}}
$$

onde: $y_{i}$ e $y_{i+1}$ são os valores de crescimento da colônia observados em duas avaliações consecutivas e $\mathrm{d}_{\mathrm{ti}} \mathrm{o}$ intervalo entre as avaliações e,

- Percentagem da inibição do crescimento micelial (ICM) calculada pela fórmula de Abbott (2): Determinada por meio da Eq. (2).

$$
\operatorname{ICM}(\%)=(T-t)^{*} 100 / T
$$

onde:

$\mathrm{T}$ é a testemunha; t o tratamento, conforme Medeiros et al. (14).

Para efeito de análise, os dados originais da ICM foram transformados em arcsen raiz $(\mathrm{x}+0,5 / 100)$ e submetidos à análise de variância e as médias comparadas pelo teste de Tukey, ao nível de 5\% de probabilidade.

Experimento 2: Efeito dos materiais orgânicos adicionados ao solo sobre o crescimento micelial do Scytalidium lignicola $\mathbf{C M M}$ 1098.

O solo utilizado neste experimento foi coletado em área proveniente de mata nativa, cuja análise química apresentou $\mathrm{pH}$ 4,5, P (16,6 mg $\left.\mathrm{Kg}^{-1}\right), \mathrm{Mg}\left(0,8 \mathrm{cMolc} \mathrm{dm}^{-3}\right), \mathrm{Ca}\left(0,8 \mathrm{cMolc} \mathrm{dm}^{-3}\right), \mathrm{Al}\left(0,15 \mathrm{cMolc} \mathrm{dm}^{-3}\right)$, $\mathrm{Na}\left(0,28 \mathrm{cMolc} \mathrm{Kg}^{-1}\right), \mathrm{K}\left(0,15 \mathrm{cMolc} \mathrm{Kg}^{-1}\right)$ e $\mathrm{H}+\mathrm{Al}\left(1,8 \mathrm{cMolc} \mathrm{dm}{ }^{-3}\right)$ de acordo com (8). A este solo foram incorporados quatro materiais orgânicos distintos: cama de aviário, esterco caprino, esterco bovino e húmus de minhoca nas concentrações, 10, 20, 30 e 40\% (v/v), sendo essas misturas, autoclavadas ou não autoclavadas.

As misturas não autoclavadas foram colocadas no fundo das placas de Petri, sobre o qual foi adicionada uma camada de AA (agarágua). Para as misturas autoclavadas, as placas contendo a mistura foi autoclavada a $120^{\circ} \mathrm{C}, 1 \mathrm{~atm}$ a 20 minutos e em seguida, uma camada de AA foi colocada na superfície. Após $24 \mathrm{~h}$, foi transferido para o centro das placas de Pétri, contendo a mistura e o meios, um disco de $8 \mathrm{~mm}$ de meio BDA contendo micélio do patógeno $S$. lignicola. Sendo as placas mantidas em B.O.D. a temperatura de $25^{\circ} \mathrm{C} \pm 2$.

As avaliações foram realizadas diariamente, de onde foi mensurado o crescimento micelial em relação ao controle. As variáveis analisadas, o delineamento experimental e a análise estatística foram as mesmas do experimento anterior. 


\section{RESULTADO E DISCUSSÃO}

Experimento 1. Avaliação do efeito de materiais orgânicos sobre o crescimento in vitro do fungo Scytalidium lignicola CMM 1098.

As doses e fontes de matéria orgânica distintas com os extratos autoclavados ou não apresentaram diferentes respostas quanto ao crescimento de $S$. lignicola CMM1098, havendo interação entre os fatores (Figura 1).

As fontes e doses de matéria orgânica permitiram uma TCM de S. lignicola que variaram de $90 \mathrm{~mm}$ a $27,50 \mathrm{~mm}$, tanto nos extratos autoclavados quanto nos não autoclavados. Os menores crescimentos miceliais foram obtidos nas placas submetidas à fonte cama de aviário, com os extratos não autoclavados, nas doses a partir de 30\% (Figura 1).

Os tratamentos contendo a dose $40 \%$ da cama de aviário não autoclavado foi o que apresentou menor taxa de crescimento micelial (Figura 1), isso acontece por causa da relação $\mathrm{C} / \mathrm{N}$ do material que favorece o crescimento de outros micro-organismos aumentando a competitividade entre eles por nutrientes (10). Dentre esses micro-organismos pode-se citar bactérias do gênero Pseudomonas que produzem biosurfactantes com poderes antimicrobianos (4). A quantidade de nitrogênio existente na cama de aviário pode ter interferido contra no crescimento micelial do S. lignicola favorecendo a biota do solo conta o patógeno. . Em trabalho realizado Ghini et al. (9) sobre efeito de adubos nitrogenados na supressividade de solos a fitopatógenos verificaram que o uso de uréia reduz a densidade populacional de alguns fungos, assim como aconteceu no presente experimento já que a quantidade de nitrogênio na CA é significativamente maior do que em outros materiais orgânicos.

A área abaixo da curva de crescimento micelial (AACCM) de $S$. lignicola apresentaram efeitos significativos para os fatores doses e fontes de matéria orgânica (Tabela 1) tanto para os extratos autoclavados quanto para os não autoclavados.

Os valores de AACCM variaram de 144 no S. lignicola CMM 1098 submetido ao tratamento com esterco bovino à $30 \%$ até 41,25 , nos fungos submetidos à cama de aviário à $30 \%$. Assim como na variável TCM, a cama de aviário na concentração de $30 \%$ nos extratos não autoclavados foi o tratamento que foi pior no que diz respeito ao crescimento micelial do S. lignicola. Essa variável explica o comportamento do crescimento micelial fúngico durante todo o tempo de duração do experimento (22).

Diferentes doses e fontes de matéria orgânica apresentaram diferentes efeitos quanto à inibição do crescimento de S. lignicola CMM 1098, apresentando interação significativa para as doses e fontes de matéria orgânica nos extratos autoclavados e não autoclavados (Figura 2).

A fonte de matéria orgânica cama de aviário foi a mais eficiente em inibir o crescimento micelial de $\boldsymbol{S}$. lignicola CMM 1098 a partir da concentração de $30 \%$ no extrato não autoclavado. É possível que a autoclavagem tenha eliminado outros micro-organismos que iriam causar o efeito supressivo do $S$. lignicola, ou que algumas moléculas importantes tenham se desnaturado pela alta temperatura e pressão da autoclavagem. A cama de aviário também foi eficiente na supressividade de outros patógenos como Phytium spp. e Scytalidium lignicola em plantas de mandioca $(10 ; 20)$.

Experimento 2: Efeito dos materiais orgânicos adicionados ao solo arenoso sobre o crescimento micelial do Scytalidium lignicola CMM 1098

Em tratamentos em que doses e fontes de matéria orgânica foram adicionadas ao solo arenoso autoclavado ou não, o crescimento do fungo apresentou diferentes respostas, com interação significativa entre fontes e doses e autoclavado ou não para todas as variáveis quando o fungo S. lignicola CMM 1098 foi adicionado ao solo (Tabela 2 e 3 e Figura 3).

Entre as diferentes fontes de matéria orgânica e doses foi visto que todas tiveram efeito similar, porém nos tratamentos autoclavados todas as fontes, bem como as doses de matéria orgânica favoreceram o crescimento de $S$. lignicola. Da mesma forma que o experimento anterior, mesmo com a adição do solo, a competição inibida pela ação da autoclavagem pode interferir no crescimento de S. lignicola CMM 1098

Os resultados de TCM estão diferentes dos encontrados por Leoni; Ghini (13) que, avaliando o efeito de matéria orgânica, tendo como fonte

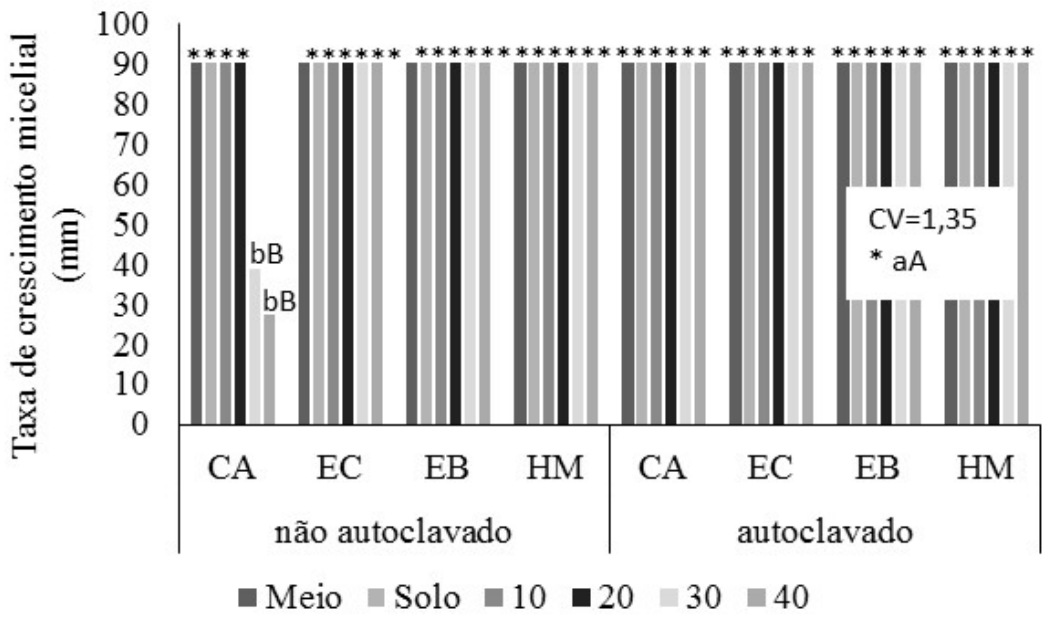

Figura 1. Taxa de crescimento micelial (em milímetros) de Scytalidium lignicola submetidos à fontes e doses de matéria orgânica, com os extratos autoclavados e não autoclavados.

*valores seguidos pela mesma letra minúscula entre as doses e maiúscula (autoclavado ou não autoclavado) não diferem estatisticamente entre si, pelo teste de Tukey, ao nível de $5 \%$ de probabilidade. $\mathrm{CA}=$ cama de aviário; $\mathrm{EC}=$ esterco caprino; $\mathrm{EB}=$ esterco bovino; $\mathrm{HM}=$ húmus de minhoca. 
Tabela 1. Área abaixo da curva de crescimento micelial Scytalidium lignicola submetidos à fontes e doses de matéria orgânica, com os extratos autoclavados e não autoclavados.

\begin{tabular}{|c|c|c|c|c|c|c|c|c|}
\hline & \multicolumn{4}{|c|}{ não autoclavado } & \multicolumn{4}{|c|}{ autoclavado } \\
\hline & CA & EC & EB & HМ & CA & EC & EB & HМ \\
\hline Meio & a 138,7 & - & - & - & - & - & - & - \\
\hline 10 & aA 131,5 & aA 124,2 & aA 120,5 & aA 129,3 & aA 129,2 & aA 131,5 & aA 142,7 & aA 139,0 \\
\hline 20 & aA 94,7 & aA 108,2 & aA 125,5 & aA 113,0 & aA 127,7 & aA 142,3 & aA 143,0 & aA 139,0 \\
\hline 40 & bB 41,2 & cB 79,7 & aA 134,2 & aA 116,8 & Aa 114,5 & aA 122,0 & aA 137,0 & aA 134,3 \\
\hline
\end{tabular}

*valores seguidos pela mesma letra minúscula na coluna e maiúscula na linha não diferem estatisticamente entre si, pelo teste de Tukey, ao nível de $5 \%$ de probabilidade. $\mathrm{CA}=$ cama de aviário; $\mathrm{EC}=$ esterco caprino; $\mathrm{EB}=$ esterco bovino; $\mathrm{HM}=$ húmus de minhoca.

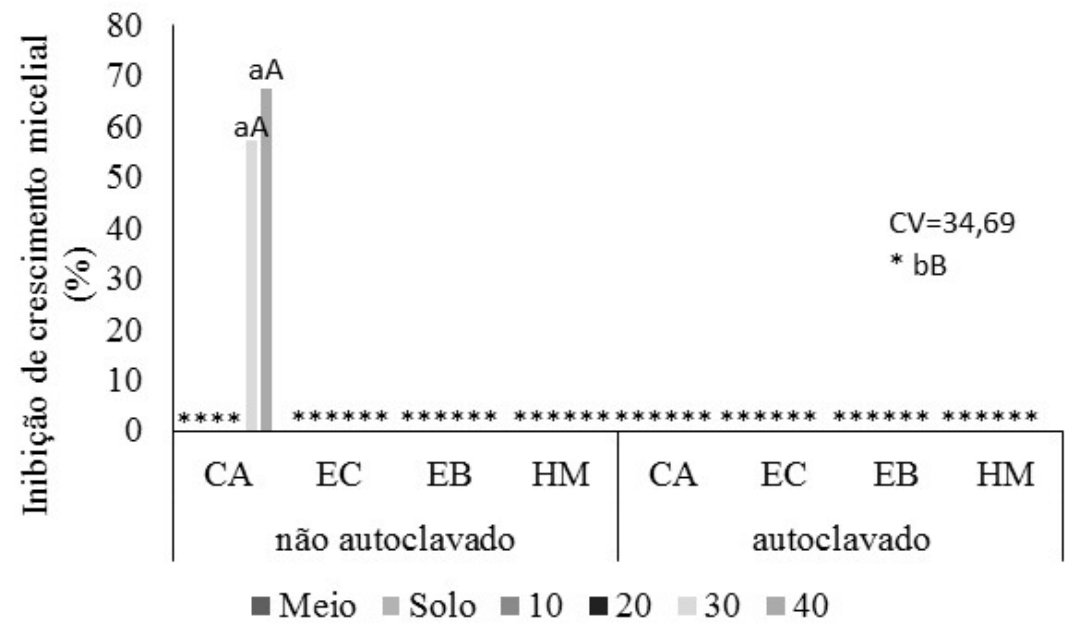

Figura 2. Inibição de crescimento micelial (em \%) de Scytalidium lignicola submetidos à fontes e doses de matéria orgânica, com os extratos autoclavados e não autoclavados.

*valores seguidos pela mesma letra minúscula entre as doses e maiúscula (autoclavado ou não autoclavado) não diferem estatisticamente entre si, pelo teste de Tukey, ao nível de 5\% de probabilidade. $\mathrm{CA}=$ cama de aviário; $\mathrm{EC}=$ esterco caprino; $\mathrm{EB}=$ esterco bovino; $\mathrm{HM}=$ húmus de minhoca

Tabela 2. Taxa de crescimento micelial (em milímetro) de Scytalidium lignicola submetidos à diferentes fontes e doses de matéria orgânica, adicionadas à solo, sendo a mistura autoclavadas e não autoclavadas.

\begin{tabular}{ccccccccccc}
\hline & \multicolumn{2}{c}{ não autoclavado } & & \multicolumn{2}{c}{ autoclavado } \\
\hline Meio & CA & EC & EB & HM & CA & EC & EB & HM \\
Solo & bA 0 & aA 90 & aA 90 & aA 90 & aA 90 & aA 90 & aA 90 & aA 90 \\
10 & bB 0 & bB 0 & bA 0 & bA 0 & bA 0 & bA 0 & bA 0 & bA 0 \\
20 & bB 0 & bB 0 & bB 0 & bB 0 & aA 90 & aA 90 & aA 90 & aA 90 \\
30 & bB 0 & bB 0 & bB 0 & bB 0 & aA 90 & aA 90 & aA 90 & aA 90 \\
40 & bB 0 & bB 0 & bB 0 & bB 0 & aA 90 & aA 90 & aA 90 & aA 90 \\
\hline
\end{tabular}

*valores seguidos pelas mesmas letras não diferem estatisticamente entre si, pelo teste de Tukey, ao nível de 5\% de probabilidade. $\mathrm{CA}=$ cama de aviário; EC= esterco caprino; $\mathrm{EB}=$ esterco bovino; $\mathrm{HM}=$ húmus de minhoca.

lodo de esgoto na indução de supressividade in vitro a Phytophthora nicotianae, observaram que quanto maior a proporção do lodo de esgoto maior foi o efeito supressivo ao fungo.

No presente estudo, houve a supressividade do crescimento micelial de $S$. lignicola submetido à tratamentos com solo usado de forma isolada (tratamento controle), assim como em mistura com diferentes fontes e doses de matéria orgânica. Indicando dessa forma que o solo está fortemente envolvido na supressividade do crescimento micelial de S. lignicola.

Outro fator que pode ter levado a esses resultados é a supressividade natural de solos na qual possuem atributos químicos, físicos, microbianos e bioquímicos que, quando não autoclavados 


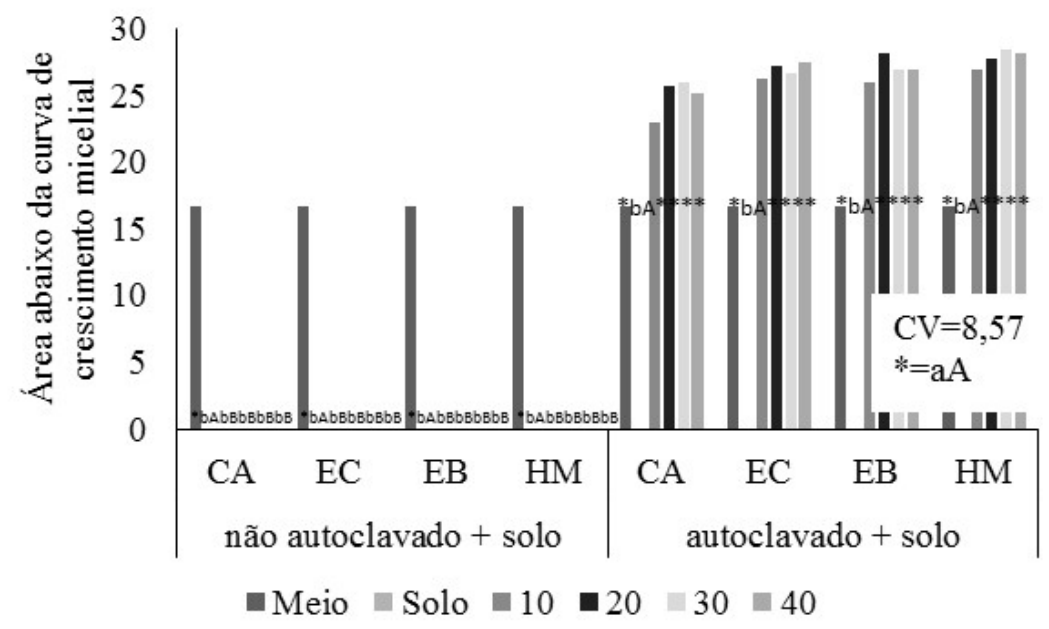

Figura 3. Área abaixo da curva de crescimento micelial (AACCM) de Scytalidium lignicola submetidos à diferentes fontes e doses de matéria orgânica, adicionadas à solos, sendo a mistura autoclavadas e não autoclavadas.

*valores seguidos pelas mesmas letras não diferem estatisticamente entre si, pelo teste de Tukey, ao nível de 5\% de probabilidade.CA= cama de aviário; $\mathrm{EC}=$ esterco caprino; $\mathrm{EB}=$ esterco bovino; $\mathrm{HM}=$ húmus de minhoca.

Tabela 3. Inibição do crescimento micelial (em \%) de Scytalidium lignicola submetidos à diferentes fontes e doses de matéria orgânica, adicionadas à solos, sendo a mistura autoclavadas e não autoclavadas.

\begin{tabular}{|c|c|c|c|c|c|c|c|c|}
\hline & \multicolumn{4}{|c|}{ não autoclavado } & \multicolumn{4}{|c|}{ Autoclavado } \\
\hline & CA & EC & EB & HМ & CA & EC & EB & HМ \\
\hline Meio & $0 \mathrm{bA}$ & $0 \mathrm{bA}$ & $0 \mathrm{bA}$ & $0 \mathrm{bA}$ & $0 \mathrm{bA}$ & $0 \mathrm{bA}$ & $0 \mathrm{bA}$ & $0 \mathrm{bA}$ \\
\hline 10 & $100 \mathrm{aA}$ & $100 \mathrm{aA}$ & $100 \mathrm{aA}$ & $100 \mathrm{aA}$ & $0 \mathrm{bB}$ & $0 \mathrm{bB}$ & $0 \mathrm{bB}$ & $0 \mathrm{bB}$ \\
\hline 20 & $100 \mathrm{aA}$ & $100 \mathrm{aA}$ & $100 \mathrm{aA}$ & $100 \mathrm{aA}$ & $0 \mathrm{bB}$ & $0 \mathrm{bB}$ & $0 \mathrm{bB}$ & $0 \mathrm{bB}$ \\
\hline 40 & $100 \mathrm{aA}$ & $100 \mathrm{aA}$ & $100 \mathrm{aA}$ & $100 \mathrm{aA}$ & $0 \mathrm{bB}$ & $0 \mathrm{bB}$ & $0 \mathrm{bB}$ & $0 \mathrm{bB}$ \\
\hline
\end{tabular}

*valores seguidos pelas mesmas letras não diferem estatisticamente entre si, pelo teste de Tukey, ao nível de 5\% de probabilidade.CA= cama de aviário; EC= esterco caprino; $\mathrm{EB}=$ esterco bovino; $\mathrm{HM}=$ húmus de minhoca.

podem interferir em diferentes estratégias para a não dominância de apenas um fungo. Como as fontes de matéria orgânica foram misturadas com solo o efeito supressivo deste pode ter ajudado no controle do desenvolvimento do fungo.

Interações complexas entre fatores abióticos e bióticos podem conduzir a supressividade natural do solo, propriedade que tem despertado muito interesse, como uma prática alternativa no controle de patógenos de vários hospedeiros. Barros et al. (3) avaliou solos arenosos com diferentes coberturas vegetais sobre a supressividade da podridão radicular da mandioca, causada por Fusarium solani e concluíram que alguns fatores bióticos dos solos foram os maiores responsáveis pelo poder supressivo, como população de bactérias totais, bactérias do grupo fluorescente, fosfatases (enzima envolvida no ciclo do $\mathrm{P}$ ) e $\beta$-glucosidase (enzima envolvida no ciclo do C). Aqui, foi utilizado solo proveniente de mata nativa e, quando adicionada matéria orgânica, pode ter contribuído para o desenvolvimento de microrganismos benéficos que não permitiram o desenvolvimento do patógeno.

A ICM de S. lignicola CMM 1098 foi maior nos tratamentos contendo solo, tanto isoladamente (controle), quanto quando misturado à fontes e doses de matéria orgânica (Tabela 3), entretanto, só teve sua máxima eficiência nos tratamentos não autoclavados.
Atualmente, diversos trabalhos utilizam solos não autoclavados em experimentos com fitopatógenos $(6 ; 7)$, pois muitos fatores ecológicos são deixados de lado quando o solo é autoclavado, como as interações entre diferentes organismos, as atividades enzimáticas que são desnaturadas quando em altas temperaturas e porque no ambiente não será encontrado solo autoclavado.

Além disso, o uso de matéria orgânica visando a supressão de um fitopatógeno vem sendo utilizada em diversos estudos e com efeitos positivos contra $R$. solani, Verticillium dahliae, Phytophthora nicotianae, Phytophthora cinnamomi, Fusarium oxysporum $f$. sp. Lini and Scytalidium lignicola $(21 ; 20)$. Além de introduzir nutrientes, micro-organismos que irão interagir positiva ou negativamente com o fitopatógeno, a aplicação de fontes de matéria orgânica podem induzir a produção de compostos tóxicos por agentes antagonistas contra os patogênicos, exercendo competição entre eles (3).

Cama de aviário não autoclavado nas doses igual ou superior a $30 \%$ foi eficiente na supressividade do crescimento micelial de $S$. lignicola CMM 1098.

Solo arenoso não autoclavados adicionado à fontes e doses de matéria orgânica são altamente eficientes na supressividade do crescimento de Scytalidium linicola CMM 1098. 


\section{AGRADECIMENTOS}

Os autores agradecem ao CNPq e à Fundação de Amparo de Ciência e Tecnologia do Estado de Pernambuco FACEPE pelo apoio financeiro (APQ-1077-5.01/10) e pelas bolsas concedidas (306401/2015-0) ao CNPq (313174/2018-0; 426497/2018-0. "O presente trabalho foi realizado com apoio da Coordenação de Aperfeiçoamento de Pessoal de Nível Superior - Brasil (CAPES)

\section{REFERÊNCIAS}

1. Anyanwu, C.N.; Ibeto, C.N.; Ezeoha, S.L.; Ogbuagu, N.J. Sustainability of cassava (Manihot esculenta Crantz) as industrial feedstock, energy and food crop in Nigeria. Renewable Energy, Brighton, v.81, p.745-752, 2015.

2. Abbott, W.S. A method of computing the effectiveness of an insecticide. Journal of Economic Entomology, Lanham, v.18, p.265-267, 1925.

3. Barros, J.A.; Medeiros, E.V.; Notaro, K.A.; Moraes, W.S.; Silva, J.E.M.; Nascimento, T.C.; Moreira, K.A. Different cover promote sandy soil suppressiveness to root rot disease of cassava caused by Fusarium solani. African Journal of Microbiology Research, Lagos, v.8, n.10, p.967-973, 2014.

4. Botelho, G.R.; Xavier, G.R.; Neves, M.C.P.; Rumjanek, N.G. A Importância dos Antibióticos Produzidos por Pseudomonas fluorescentes na Supressão de Doenças de Plantas. Seropédica: Embrapa Agrobiologia, 2006. 31p. (Embrapa Agrobiologia. Documentos, 211).

5. Buensanteai, N.; Athinuwat, D. The antagonistic activity of Trichoderma virens strain TvSUT10 against cassava stem rot in Thailand. African Journal of Biotechnology, Nairobi, v.11, n.84, p.14996-15001, 2012.

6. Da Silva, J.A.T.; De Medeiros, E.V.; Da Silva, J.M.; Tenório, D.D.A.; Moreira, K.A.; Nascimento, T.C.E.D.S.; Souza $\square$ Motta, C. Trichoderma aureoviride URM 5158 and Trichoderma hamatum URM 6656 are biocontrol agents that act against cassava root rot through different mechanisms. Journal of Phytopathology, Göttingen, v.164, n.11/12, p.1003-1011, 2016.

7. Da Silva, J.A.T.; De Medeiros, E.V.; Da Silva, J.M.; Tenório, D.D.A.; Moreira, K.A.; Nascimento, T.C.E.D.S.; Souza $\square$ Motta, C. Antagonistic activity of Trichoderma spp. against Scytalidium lignicola CMM 1098 and antioxidant enzymatic activity in cassava. Phytoparasitica, Rehovotv, v.45, n.2, p.219-225, 2017

8. Embrapa. Manual de análises químicas de solos, plantas e fertilizantes. Brasília, DF: Embrapa Informação Tecnológica, 2009. 627p.

9. Ghini, R.; Bettiol, W.; Dynia, J.F.; Maia, A.H.N. Efeito de Adubos Nitrogenados na Supressividade de Solos a Fitopatógenos. Ecossistema, Espírito Santo do Pinhal, v.26, n.2, p. 147-151, 2001

10. Ghini, R.; Schoenmaker, I.A.S.; Bettiol, W. Solarização do solo e incorpoe ração de fontes de matéria orgânica no controle de Pythium spp. Pesquisa
Agropecuária Brasileira, Brasília, v.37, n.9, p.1253-1261, 2002.

11. Hoitink, H.A.J.; Boehm, M.J. Biocontrol within the context of soil microi bial communities: a substrate dependent phenomenon. Annual Review of Phytopathology, Palo Alto, v.37, p.427-446, 1999.

12. Kasuya, M.; Olivier, A.R.; Ota, Y.; Tojo, M.; Honjo, H.; Fukui, R. Induction of Soil Suppressiveness Against Rhizoctonia solani by Incorporation of Dried Plant Residues into Soil. Phytopathology, Saint Paul, v.96, n.12, p.1372-1379, 2006

13. Leoni, C.; Ghini, R. Efeito do lodo de esgoto na indução de supressividade in vitro a Phytophthora nicotianae. Fitopatologia Brasileira, Fortaleza, v.28, p.67-75, 2003

14. Medeiros, E.V.; Viana, M.G.; Albuquerque, C.C.; Viana, F.A.; Silva, K.M.B. Extrato etanólico de Senna alata no controle de Fusarium oxysporum, causador da murcha-de-fusarium do meloeiro. Revista Brasileira de Engenharia Agrícola e Ambiental, Campina Grande, v.16, n.11, p.1166-1170, 2012.

15. Montagne, V.; Capiaux, H.; Cannavo, P.; Charpentier, S.; Renaud, S.; Liatard, E; Lebeau, T. Protective effect of organic substrates against soil-borne pathogens in soilless cucumber crops. Scientia Horticulturae, Amsterdam, v.206, p.62-70, 2016.

16. Muniz, M.F.S.; Andrade, F.W.R.; Queiroz, F.M.; Moura Filho, G.; Menezes, M. Caracterização de isolados de Phytophthora drechsleri, agente causal da podridão mole de raízes de mandioca. Tropical Plant Pathology, Lavras, v.31, p.195-198, 2006.

17. Notaro, K.A.; Medeiros, E.V.; Silva, C.A.D.; Barros, J.A. Prospecção de fitopatógenos associados á podridão radicular da mandioca em Pernambuco, Brasil. Bioscience Journal, Uberlândia, v.29, n.6, p. 1832-1839, 2013.

18. Oliveira, M.A.; Fiorine, R.A. Análise de crescimento em mudas de mandioca (Manihot esculenta Crantz) provenientes de estacas em diferentes recipientes para cultivo. Revista Raízes e Amidos Tropicais, Botucatu, v.2, p.12-26, 2006.

19. Onyeka, T.J.; Dixon, A.G.O.; Ekpo, E.J.A. Field evaluation of root rot disease and relationship between disease severity and yield in cassava. Experimental Agriculture, Cambridge, v.41, p.357-363, 2005

20. Silva, C.A.D.; Medeiros, E.V.; Bezerra, C.B.; Morais Silva, W.; Barros, J.A.; Santos, U.J. Interferência da incorporação de matéria orgânica no solo no controle da podridão negra da mandioca, causada por Scytalidium lignicola. Bioscience Journal, Uberlândia, v.29, n.6, p. 1823-1831, 2013.

21. Termorshuizen, A.J.; Van Rijn, E.; Van Der Gaag, D.J.; Alabouvette, C.; Chen, Y.; Lagerlof, J.; Malandrakis, A.A.; Paplomatas, E.J.; Ramert, B.; Ryckeboer, J.; Steinberg, C.; Zmora-Nahum, S. Suppressiveness of 18 composts against 7 pathosystems: variability in pathogen response. Soil Biology and Biochemistry, Elmsford, v.38, n.8, p.2461-2477, 2006.

22. Viana, M.G.; Albuquerque, C.C.; Medeiros, E.V.; Viana, F.A.; Silva, K.M.B. Avaliação do potencial fungicida de extratos etanólicos de Senna alata contra Monosporascus cannonballus. Ciência e Agrotecnologia, Lavras, v.32, n.5, p.1387-1393, 2008 